\title{
A Note on Categorial Grammar, Disharmony and Permutation
}

\author{
Crit Cremers \\ Leiden University, Department of General Linguistics \\ P.O. box 9515, 2300 RA Leiden, The Netherlands \\ cremers@rullet.leidenuniv.nl
}

Disharmonious Composition (DishComp) is definable as

$\mathrm{X} / \mathrm{Y} \mathbf{Y} \backslash \mathbf{Z} \Rightarrow \mathbf{X} \backslash \mathbf{Z} \quad \mathrm{Y} / \mathrm{Z} \mathbf{X} \backslash \mathbf{Y} \Rightarrow \mathrm{X} / \mathbf{Z}$

(and is comdemned by Carpenter 1998: 202

and Jacobson 1992: 139ff)

Harmonious Composition (HarmComp) is defined as

$\mathrm{X} / \mathrm{Y} \mathrm{Y} / \mathrm{Z} \Rightarrow \mathrm{X} / \mathrm{Z} \quad \mathrm{Y} \backslash \mathrm{Z} \mathbf{X} \backslash \mathrm{Y} \Rightarrow \mathrm{X} \backslash \mathrm{Z}$

(and is generally adored)

Lambek Calculus (Lambek) has the following basis:

axiom: $\mathbf{X} \Rightarrow \mathbf{X}$

rules: if $\mathbf{X} \mathbf{Y} \Rightarrow \mathbf{Z}$ then $\mathbf{X} \Rightarrow \mathbf{Z} / \mathbf{Y}$

and $\mathrm{Y} \Rightarrow \mathrm{Z} \backslash \mathrm{X}$

if $\mathrm{X} \Rightarrow \mathrm{Z} / \mathrm{Y}$ then $\mathrm{X} \mathrm{Y} \Rightarrow \mathrm{Z}$

if $\mathbf{X} \Rightarrow \mathbf{Z} \backslash \mathbf{Y}$ then $\mathbf{Y} \mathbf{X} \Rightarrow \mathbf{Z}$

Permutation Closure of language L (PermL)

PermL $=\left\{s \mid s^{\prime}\right.$ in $\mathrm{L}$ and $s$ is a permutation of $s\}$ and $\mathrm{L} \subseteq$ PermL

(but nice languages are not PermL for any $L$ )

Fact 1

DishComp is not a theorem of Lambek but HarmComp is

(as you can easily check)

Fact 2

DishComp + Lambek $=$ Lambek + Permutation $=$ undirected Lambek (Moortgat 1988, Van Benthem 1991; Lambek is maximal, but contextfree)

$\Leftrightarrow$

For any assignment $\mathrm{A}$ of categorial types to the atoms of language $L$, if Lambek recognizes
L under A, Lambek + DishComp recognizes PermL under $A$

(so disharmony is always too much for Lambek)

Generalized Composition (GenComp) (Joshi et al. 1991, Steedman 1990)

primary type secondary type composition $\mathbf{X} / \mathbf{Y} \quad\left(. .\left(\mathbf{Y} \mid \mathbf{Z}_{1}\right) ..\right)\left|\mathbf{Z}_{\mathrm{n}} \Rightarrow\left(. .\left(\mathbf{X} \mid \mathbf{Z}_{1}\right) ..\right)\right| \mathbf{Z}_{\mathrm{n}}$ secondary type primary type composition $\left(. .\left(\mathbf{Y} \mid \mathbf{Z}_{1}\right) ..\right)\left|\mathbf{Z}_{\mathrm{n}} \quad \mathbf{X}\right| \mathbf{Y} \quad \Rightarrow\left(. .\left(\mathbf{X} \mid \mathbf{Z}_{1}\right) ..\right) \mid \mathbf{Z}_{\mathrm{n}}$ while $\mid$ is $\backslash$ or $/$ and is conserved under composition.

(Summarizing combinatory categorial grammar:)

Fact 3

GenComp entails DishComp

(and you need it for the famous crossing dependencies in Dutch, but)

\section{Fact 4}

It is not the case that for any assignment $\mathrm{A}$ of categorial types to the atoms of language $L$, if GenComp recognizes $L$ with respect to A, GenComp recognizes PermL with respect to $\mathrm{A}$

(as you can see from:)

$\underline{\mathrm{MIX}}$

MIX $=$ PermTRIPLE, where TRIPLE $=$ $\left\{\mathrm{a}^{\mathrm{n}} \mathrm{b}^{\mathrm{n}} \mathrm{c}^{\mathrm{n}}: \mathrm{n}>0\right\}$

(- which is more than mildly context-sensitive; Joshi et al. 1991 - and)

\section{Fact 5}

Consider the assignment $A_{b}$ of categories to the lexicon $\{a, b, c\}$ s.t. $A_{b}(a)=a$, $A_{b}(c)=c, A_{b}(b)=\{(s / a) / c,((s / a) / c) / s$, 
$\ldots,((s \backslash c) / s) \backslash a, \ldots((s \backslash s) \backslash c) \backslash a,(s \backslash c) \backslash a\}$, i.e. $\mathrm{A}_{\mathrm{b}}(\mathrm{b})=\{\mathrm{s}|\mathrm{x}| \mathrm{y}, \mathrm{s}|\mathrm{v}| \mathrm{w}|\mathrm{t}|\{\mathrm{x}, \mathrm{y}\}=\{\mathrm{a}, \mathrm{b}\}$, $\{\mathrm{v}, \mathrm{w}, \mathrm{t}\}=\{\mathrm{a}, \mathrm{c}, \mathrm{s}\}$ and $\mid$ is $\backslash$ or $/\} ; \mathrm{b}$, then, is said to be fully functional, since it has all relevant functional types.

GenComp does not recognize MIX with respect to assignment $\mathbf{A}_{\mathbf{b}}$.

For example: GenComp does not derive $b a a c c b$ and $a b a a c c b c b$ with respect to $A_{b}$

\section{Fact 6}

Let $A_{b c}(a)=A_{b} a, A_{b c}(b)=A_{b}(b), A_{b c}(c)$ $=\{(s / a) / b,((s / a) / b) / s, \ldots,((s \backslash b) / s) \backslash a$, $\ldots((s \backslash s) \backslash b) \backslash a,(s \backslash b) \backslash a\}$ (both $b$ and $c$ are fully functional).

GenComp recognizes MIX with respect to assignment $A_{b c}$.

(Now consider the grammar exhibiting the following features.)

Primitive Cancellation Constraint

$\mathrm{X} / \mathrm{Y} \mathrm{Y} \Rightarrow \mathrm{X}$ iff $\mathrm{Y}$ is primitive

(- in order to be more restrictive - and)

Directed Stacks (example)

$(((\mathrm{X} \backslash \mathrm{Y}) / \mathrm{W}) \backslash \mathrm{U}) / \mathrm{V}$ is written as

$\mathrm{X} \backslash[\mathrm{U}, \mathrm{Y}] /[\mathrm{V}, \mathbf{W}]$

(- in order to be more transparent - and)

Transparent Primary Category (examples)

$\mathrm{X} \backslash[\mathrm{A}] /[\mathrm{Y}, \mathrm{B}] \mathrm{Y} \backslash[\mathrm{C}] /[\mathrm{D}] \Rightarrow \mathrm{X} \backslash[\mathrm{A}, \mathrm{C}] /[\mathrm{B}, \mathrm{D}]$ or

$\mathrm{X} \backslash[\mathrm{A}] /[\mathrm{Y}, \mathrm{B}] \mathrm{Y} \backslash[\mathrm{C}] /[\mathrm{D}] \Rightarrow \mathrm{X} \backslash[\mathrm{C}, \mathrm{A}] /[\mathrm{B}, \mathrm{D}]$ or

$\mathrm{X} \backslash[\mathrm{A}] /[\mathrm{Y}, \mathrm{B}] \mathrm{Y} \backslash[\mathrm{C}] /[\mathrm{D}] \Rightarrow \mathrm{X} \backslash[\mathrm{A}, \mathrm{C}] /[\mathrm{D}, \mathrm{B}]$ or

$\mathbf{X} \backslash[\mathrm{A}] /[\mathbf{Y}, \mathrm{B}] \mathrm{Y} \backslash[\mathrm{C}] /[\mathrm{D}] \Rightarrow \mathbf{X} \backslash[\mathrm{C}, \mathrm{A}] /[\mathrm{D}, \mathrm{B}]$

(- in order to gain expressivity - make Gen-

Comp into)

Categorial List Grammar (CatListGram)

(Cremers 1993 and at fonetiek-

6.leidenuniv.nl/hijzlndr/delilah.html)

GenComp + Primitive Cancellation Constraint + Directed Stacks + Transparent Primary Category

(but nevertheless)

\section{Fact 7}

Fact 4, Fact 5 and Fact 6 also hold $m u$ tatis mutandis for CatListGram. In these aspects, CatListGram and GenComp are weakly equivalent.

\section{CONCLUSIONS}

None of the additional characteristics for CatListGram affects the weak capacity of a categorial grammar; i.e.:

- exclusive cancellation of primitives does not affect recognition capacity

- maintaining more than one argument stack does not affect recognition capacity

- merging argument stacks of primary and secondary category does not affect recognition capacity

and it takes more than disharmony to induce permutation closure.

\section{References}

Benthem, J. van, Language in Action, North Holland, 1991

Carpenter, B., Type-Logical Semantics, MIT Press, 1997

Cremers, C., On Parsing Coordination Categorially, HIL diss, Leiden University, 1993 Jacobson, P., 'Comment Flexible Categorial Grammars', in: R. Levine (ed.), Formal grammar: theory and implementation, Oxford Univ. Press, 1991, p. $129-167$

Joshi, A.K., K. Vijay-Shanker, D. Weir, 'The Convergence of Mildly Context-Sensitive Grammar Formalisms', in: P. Sells, S.M. Shieber, T. Wasow (eds), Foundational Issues in Natural Language Processing, MIT Press, 1991, pp. $31-82$

Moortgat, M., Categorial Investigations, Foris, 1988

Steedman, M., 'Gapping as Constituent Coordination', Linguistics and Philosophy 13, p. $207-263$ 